\title{
El influencer de moda como sujeto de relaciones públicas:
}

\section{recursos y herramientas}

\author{
Villena Alarcón, Eduardo \\ Universidad de Málaga (UMA) \\ eduardo.villena@uma.es
}

Forma de citar este artículo: Villena, E. (2018). El Influencer de moda como sujeto de relaciones públicas: recursos y herramientas, Redmarka. Revista de Marketing Aplicado, vol 01, núm. 022, 115-128. https://doi.org/10.17979/redma.2018.01.022.4938

\section{Resumen:}

Internet y las redes sociales han favorecido la aparición de un nuevo perfil profesional dentro del mundo de la moda: el influencer. Un gran número de empresas han incorporado ya a sus estrategias de comunicación las relaciones con estos nuevos líderes de opinión; los cuales se han revelado como auténticos prescriptores de las firmas de moda. Sin embargo, son exiguos los estudios que ponen de manifiesto la importancia de esta nueva figura y la necesidad de identificarlos correctamente. Por ello, la consiguiente investigación propone una revisión documental que permita dirimir el verdadero valor de las acciones con influencers como estrategia de relaciones públicas 4.0.; así como determinar cuáles son los recursos y las herramientas más importantes para lograr seleccionar quiénes más nos convengan. De los resultados arrojados por la investigación podemos dilucidar una necesidad por parte de las relaciones públicas de aportar un valor estratégico a esta actividad. Concluye un documento que aporta una hoja de ruta por recursos y herramientas que permite a los directores de comunicación identificar correctamente a estos nuevos colaboradores en función de sus necesidades y objetivos. 
Palabras clave: Influencers, relaciones públicas 4.0., moda.

\begin{abstract}
:
Internet and social networks have favoured the emergence of a new professional profile within the world of fashion: the influencer. A great number of companies have already incorporated relations with influencers into their communication strategies. However, It is important to emphasize that the studies related to the relevant issue are not enough and they do not show the value of this new figure. For this reason, this research proposes a literature review that allows to determine the real value of the actions with influencers as a 4.0 public relations strategy; as well as to determine which are the most important resources and tools and what are the most suitable for us. In view of the results obtained in this research, a need on the part of the public relations to contribute a strategic value to this activity can be clarified. This paper provides a roadmap for resources and tools that enables communication managers to identify these new collaborators based on their needs and objectives.
\end{abstract}

Keywords: Influencer, 4.0 public relations, fashion.

\title{
1. INTRODUCCIÓN
}

La rápida evolución de Internet y el gran factor de innovación que define actualmente las tecnologías de la comunicación y la información ha generado una transformación de la disciplina hacia las relaciones públicas 4.0.; lo que ha provocado el surgimiento de nuevos perfiles profesionales llamados a cubrir las nuevas necesidades de los stakeholders de las organizaciones. Entre las recientes profesiones que definen el ámbito que nos ocupa se encuentran los influencers, personas con gran capacidad de prescripción capaces de atraer a nuevos públicos a las empresas de moda.

La concurrencia de esta figura en el proceso comunicativo de las firmas ha favorecido la aparición de nuevas estrategias de relaciones públicas capaces de poner en práctica innovadoras formas de provocar engagement. Con ello, y gracias a los avances de las diferentes herramientas de las que se dispone, se ha logrado que el mensaje llegue de manera más eficiente a los destinatarios. Si 
atendemos a las rutinas más habituales, nos percatamos de que, a pesar del crecimiento de la industria, la mayoría de colaboraciones con influencers sigue estando al $1 \%$, a pesar de que refuerza la marca y permite alcanzar nuevas audiencias ${ }^{1}$.

Especial mención merecen los microinfluencers que, a pesar de generar un impacto ecuánime a su influencia, su engagement es superior al de aquellos influencers con más seguidores. Retomando lo expuesto por Díaz (2017), si consideramos que en un prescriptor ha de preponderar su poder de prescripción sobre el de generar conocimiento, es más efectivo dirigir acciones a nicho a través de un influencer con menor número de seguidores pero con mayor influencia sobre los mismo, que a través de uno con gran número de seguidores, pero con menor poder de influencia.

Su eficiencia en el mercado actual es manifiesta y el retorno de la inversión está asegurado. Además de que existen tantos influenciadores como el sector exija, su contenido no puede bloquearse y los públicos siguen recurriendo a las recomendaciones para generar confianza. De igual forma, el alto compromiso y los bajos costes de estas campañas ayudan a las marcas a dinamizar sus audiencias y obtener cientos de nuevos seguidores por cada acción. La confianza se revela pues aquí como uno de los intangibles más apreciados y completos para las firmas de moda, las cuales demandan estrategias de comunicación más específicas y complejas (Viñarás, 2015).

Por otro lado, son escasas las contribuciones realizadas desde la Academia acerca de la comunicación con influencers en el sector de la moda; no obstante, cabe destacar lo aportado por Castelló (2015) ó Del Río (2011) sobre prescriptores y redes sociales; ó Violante y Laurent (2017) sobre nuevos perfiles profesionales. Desde la perspectiva profesional, el mercado demanda profesionales que sean capaces tanto de identificar a los líderes de opinión con comunidades que sean de interés para las marcas, como de gestionar estrategias específicas para tal empresa (Illera y Monge, 2018). Por ello, a través

\footnotetext{
${ }^{1}$ El influencer marketing será en 2018 un sano y eficiente marketplace (16 de enero de 2018). Marketing Directo. Recuperdo de: https://goo.gl/eHxsdc
} 
de las consiguientes líneas, este artículo propone una revisión documental que permita dirimir el verdadero valor de las acciones con influencers como estrategia de relaciones públicas, así como determinar cuáles son los recursos y las herramientas más importantes para lograr seleccionar a los prescriptores más pertinentes.

\section{METODOLOGÍA}

El artículo acude a la revisión bibliográfica y evidencia la importancia de este tipo de actividades para la reflexión de la propia práctica pedagógica (González y Barba, 2013) y profesional. Como método cualitativo, a través de la revisión bibliográfica se han planteado como premisas de partida una serie de preguntas de investigación que orientan la consecución del texto:

- ¿Cómo se define el influencer de moda como sujeto de relaciones públicas 4.0 ?

- ¿Qué lugar ocupa el líder de opinión dentro de la estrategia de relaciones públicas $4.0 ?$

- ¿Cuáles son los recursos y las herramientas específicas para las relaciones con los influencers?

El objetivo principal de este estudio es proponer recursos y herramientas propias de las relaciones públicas 4.0. encaminadas a la correcta gestión de las relaciones con los influencers por parte de los profesionales del sector. Para ello, se sigue como método el estudio bibliográfico del concepto de influencer como profesional de las relaciones públicas, tomando como referencia lo postulado acerca de él en materia de comunicación, relaciones públicas y estrategia.

\section{EL INFLUENCER DE MODA COMO SUJETO DE RELACIONES PÚBLICAS 4.0.}

La moda es un lugar de constante cambio (Katz y Lazarsfeld, 2006) donde las relaciones interpersonales son muy relevantes (Sabada y San Miguel, 2014). En este escenario, los profesionales de las relaciones públicas en la moda han encontrado una oportunidad única para implementar nuevas herramientas de 
comunicación a través de la figura de los influencers. Como introito al concepto, podemos considerar lo aportado por Bongaga y Turiel (2016) cuando afirman que los influencers son personas que, gracias a su carisma y a sus capacidades de comunicación y de conectar con el público, han alcanzado una fama online que les ha hecho amasar una legión de seguidores en la red. $O$ en otras palabras, alguien que, por su personalidad, genera influencia en otros consumidores por sus opiniones, valoraciones y decisiones de compra (Castelló y del Pino, 2015). De cualquier forma, los relacionadores públicos se encuentran ante una nueva figura profesional de reconocida reputación digital que, como expertos en una determinada materia, en este caso la moda, publican contenidos que son leídos por miles de seguidores y generan una comunidad a su alrededor con elevadas tasas de interacción y engagement (Castelló Martínez, 2016). Según Capriotti (2013), el influencer se alza a partir de la necesidad de identificar prescriptores en las redes sociales gracias a su estatus o a su conocimiento.

Ya de manera específica, Díaz (2017) lista en tres tipos la consiguiente figura en función de su capacidad de prescripción: celebrity influencer, social media influencer y los microinfluencer. De entre todos, el personaje famoso tiene la prescripción más baja a pesar de acceder a un número de público mayor; sin embargo, los social media influencers y los microinfluencers ${ }^{2}$ tienen más capacidad para influir en el entorno cercano a un menor coste. De hecho, los microinfluencers se han convertido en un elemento cada vez más interesante dentro de las relaciones públicas 4.0. porque permite llegar a públicos más concretos y específicos, y permiten conectar con ellos con mayor grado de engagement.

Igualmente, entre las múltiples oportunidades que ofrece este perfil profesional a las relaciones públicas en empresa de moda se encuentra la posibilidad de conocer mejor a los públicos con los que interactúa la firma en los entornos sociales e identificar aquellos con un mayor nivel de influencia. Según el

\footnotetext{
${ }^{2}$ Los 5 cambios que se han implantado en 2017 en el marketing con influencers (19 de diciembre de 2017). Puro Marketing. Recuperado de: https://goo.gl/72bbNQ
} 
Informe de PwC (2016), las compañías que sean capaces de identificar, gestionar y medir sus estrategias de relaciones públicas con los influencers de su comunidad obtendrán en el futuro una ventaja competitiva frente a otras marcas de la industria.

La realidad profesional queda manifiesta en el Informe de Augure: un $81 \%$ de los profesionales encuestados afirmaron que las relaciones con influencers son eficaces a la hora de alcanzar sus objetivos. De hecho, el $70 \%$ de los consultados consideró que la participación de un influencer en un evento organizado por la marca constituye el formato más eficaz para sacar partido de su influencia. Sin embargo, su figura no está aún profesionalizada dentro de la disciplina. Prueba de ello es que solo el $35 \%$ de las grandes empresas los remuneran, frente al $29 \%$ de las medianas empresas y el $15 \%$ de las pequeñas.

De cualquier forma, son los directores de relaciones públicas los llamados a gestionar la relación con los influencers; por delante de otras figuras como el community manager y el director ejecutivo. Una situación que varía en función del tamaño de la compañía. Por otro lado, solo el $25 \%$ dispone de presupuesto específico para tal actividad y encuentran su principal desafío en identificar a los influencers más relevantes para la estrategia de la marca, así como disponer de indicadores adecuados para medir la eficacia de sus campañas implementadas. $Y$ es que, para que una campaña con influencers sea efectiva no es suficiente con identificar a los más representativos del sector; hay que ser capaces de evaluar quiénes son los se ajustan a los objetivos de nuestra campaña (ADECEC, 2016). 
Figura 1: Herramientas eficaces para trabajar con influencers

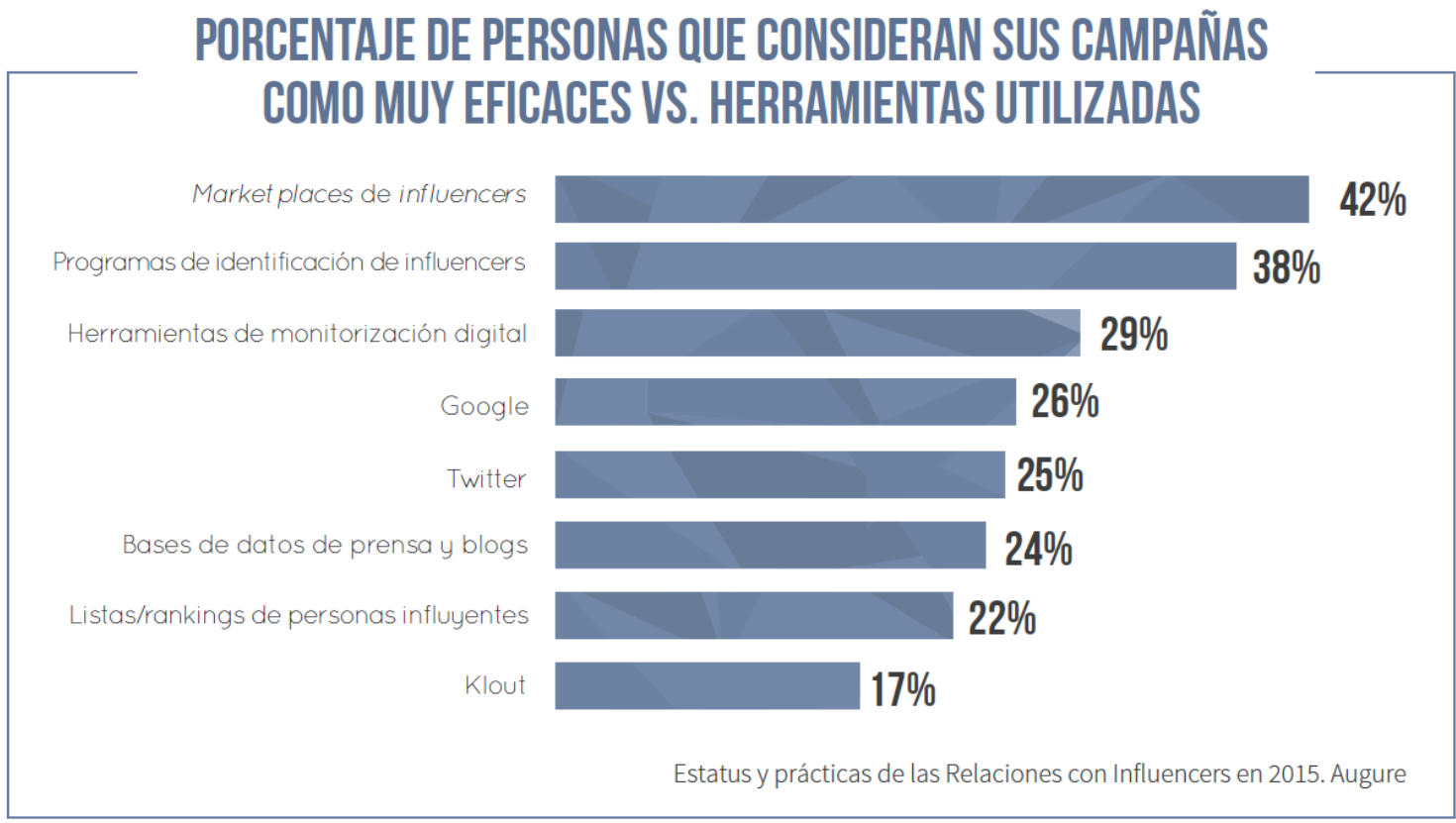

Fuente: Augure

Finalmente, a todo ello hay que unirle que las plataformas más utilizadas para gestionar la relación con los influencers no siempre son las más eficaces. Tal y como se puede observar en el gráfico 1, quiénes utilizan plataformas especializadas para gestionar las relaciones con estos prescriptores realizan campañas más eficaces que las que utilizan redes sociales.

\section{RECURSOS Y HERRAMIENTAS PARA LAS RELACIONES CON INFLUENCERS DE MODA}

La eficiencia que representa ha situado las relaciones con los influencer en el centro de cualquier actividad de relaciones públicas 4.0.; sin embargo, tal y como se ha expuesto en líneas anteriores, un elemento indispensable en la planificación de campañas con prescriptores es la adecuada identificación de estos perfiles en los distintos canales. De igual forma, resulta apropiado que, a la hora de seleccionar al profesional, se tenga presente las particularidades propias que definen al líder de opinión y valorar la adecuación con la marca. ADECEC (2016) recuerda en su Informe que un buen influencer no es el que tiene más cantidad de followers. Es más, contar con multitud de seguidores no es el dato más relevante para seleccionar a uno. Sea como fuere, desde los de 
mayor influencia a los de menos, el primer paso a seguir por una empresa que quiera incorporarlos en su estrategia de relaciones públicas debe considerar a unos y a otros en su influencers map (Castelló y del Pino, 2015).

Por otro lado, al igual que en los medios convencionales, las apariciones en las plataformas propias de los influencers pueden ser tanto ganadas como pagadas, en función de la campaña y la estrategia (ADECEC, 2016). Por tanto, el documento estratégico que recoja la acción deberá, cuanto menos:

- $\quad$ fijar los objetivos de la campaña, a corto, medio y largo plazo.

- establecer el posicionamiento de marca, los valores y atributos que la hacen diferente.

- definir el mensaje que definirá la campaña.

- averiguar si los influencers ya hablan de la empresa y en qué términos.

- priorizar los públicos a los que se va a dirigir y diseñar un mapping.

Sea como fuere, algunos de los instrumentos y de las herramientas destacadas en las siguientes líneas para establecer relaciones con los prescriptores se ocupan tanto de la selección de los influencers como de la gestión y organización de las campañas o acciones planificadas con ellos.

\subsection{Audiense}

La compañía textil Puma, a través de la agencia DigitasLBi, recurrió a Audiense y su segmentación avanzada para identificar influencers locales en todo el mundo en su campaña \#IGNITEYourCity. Con ello se logró impactar sobre los públicos que tenían intereses en el running e impulsar la conversación a través de la funcionalidad de monitorización, que permitió organizar y analizar la actividad de los participantes. A día de hoy, Audiense se ha posicionado como una de las principales herramientas para identificar públicos relevantes del sector de la moda dentro de las principales redes sociales. A través de Audiense Insight se logran planificar campañas de relaciones públicas más efectivas ya que permite que las firmas puedan conectar con su target y segmentarlos, gracias a su aprendizaje automático, en función de la edad, el género, su autoridad, su 
actividad y dónde estén ubicados. La plataforma dispone también de Audiense Connect, una herramienta específica para Twitter. Entre las últimas actualizaciones se encuentra el Influencers Report, una solución de Audiense Insights que permite identificar microinfluencers para convertirse en prescriptores reales de una marca.

Del mismo modo, Klear es una herramienta similar a la anterior; de gran utilidad a la hora de identificar, analizar y medir las acciones con influencers. Dentro de su web, el usuario puede buscar prescriptores en blogs y las principales redes sociales a través de las palabras clave y la localización, al igual que acceder a analíticas con datos acerca de su presencia en redes sociales. Permite también monitorear y crear informes sus actividades, y gestionar campañas.

\subsection{Followerwonk}

En el caso de realizar campañas específicas en Twitter, el software de Followerwonk identifica las cuentas con mayor autoridad social en función de los retweet, la cantidad de tweets publicados y el número de seguidores. Resulta especialmente oportuno si el objetivo es identificar personas relevantes para la empresa de moda dentro del microentorno ya que habilita una barra de búsqueda en función de la localización y las palabras clave. De igual forma, Iconosquare, otra herramienta similar, responde en Instagram y Facebook a estas necesidades y a otras más específicas.

Por otro lado, Twitteraudit posibilita conocer el porcentaje de seguidores falsos que tiene un perfil de Twitter. Esto es interesante si queremos saber si el profesional es realmente un líder ampliamente seguido o por el contrario ha aumentado sus seguidores de manera inorgánica. Para comprobar si un usuario es real o falso se puntúa el número de tweets, la fecha del último tweet y la proporción de seguidores.

\subsection{Influencerhub}

La marca de ropa Sprinter implementó distintas acciones con influencers del sector deportivo a través de Influencerhub, un producto de la Consultora BEST que en el último año ha duplicado su plantilla e integrado nuevos servicios, 
proyectos y partners ${ }^{3}$. Sprinter identificó y contactó con más de 50 prescriptores que se identificaran con el espíritu UrbanHero; es decir, aquellas personas que corren para recoger a sus hijos y que además tienen tiempo para entrenar y vivir deportivamente ${ }^{4}$. En una clara apuesta por las relaciones públicas 4.0., Influencerhub tiene como misión la gestión de la influencia. Se trata de un espacio digital que relaciona a líderes de opinión de diversos sectores con las marcas que trabajan con la consultora ${ }^{5}$ y que persigue establecer fuertes vínculos con las personas con las que se relaciona, recabando información cualitativa de alto nivel para personalizar acciones y proyectos ${ }^{6}$. Esta herramienta permite realizar análisis de conversaciones para optimizar los procesos de escucha activa, búsqueda de tendencias y detección e identificación de perfiles cualificados ${ }^{7}$.

Teniendo como referencia este modelo de negocio para las relaciones públicas, podemos citar otras plataformas para gestionar campañas con influencers de nicho como BranTube, que une empresas de moda con los principales youtubers. Gracias a su plataforma, el usuario puede analizar a los influencers que con los que cuentan en cartera y ver cuál se adapta mejor a las necesidades de la acción.

\section{4. Índices de influencia}

Si la campaña exige conocer la capacidad de influir que un prescriptor tiene a la hora de transmitir los contenidos la solución es Klout, un servicio web que desde 2008 mide el grado de influencia de una persona o una marca en las Redes Sociales. Gracias al Klout Score se puede conocer la idoneidad de su influencia a partir de ciertos parámetros que se actualizan a diario en función de las redes sociales más importantes. El alcance, la amplificación y la red del influencer son los tres apartados fundamentales que la plataforma pone a disposición del

\footnotetext{
${ }^{3}$ La consultora de comunicación Best Relations se separa (15 de febrero 2018). Marketing Directo. Recuperado de: https://goo.gl/zk8Dhp

${ }^{4}$ Sprinter. Influencer marketing (s.f.). Recuperado de: https://goo.gl/VHL5Jy

${ }^{5}$ Qué nuevos servicios ofrecen las agencias de comunicación a sus clientes. (10 de enero de 2018). Top comunicación y RRPP. Recuperado de: https://goo.gl/NQB1U2

${ }^{6}$ La plataforma digital Brandchats se une a Best Relations. (4 de octubre de 2017). Recuperado de: https://goo.gl/yVZDqP

${ }^{7}$ Qué nuevos servicios ofrecen las agencias de comunicación a sus clientes. (10 de enero de 2018). Top comunicación y RRPP. Recuperado de: https://goo.gl/NQB1U2
} 
usuario. Diferentes empresas de moda promocionan ya de forma exclusiva sus productos a través de los influencers que tengan un Klout Score elevado. De hecho, Gilt Groupe, una web de compras de lujo, ofreció descuentos en proporción al Klout de cada cliente ${ }^{8}$.

Kred es otro servicio online que se encarga de medir la influencia de un usuario en las redes sociales y así poder identificar a las personas influyentes en las diferentes comunidades basadas en intereses y sectores. Parecida a Klout, Kred es una herramienta que permite evaluar la influencia en las RRSS a través de un algoritmo que consiste en dos tipos de factores: el número de usuarios que reaccionan ante un post y el alcance del mismo, que se mide partiendo del número de usuarios al que llega el post.

\subsection{Buzzsumo}

Desde la perspectiva del contenido, podemos identificar influencers en función del grado en el que ha sido compartido. Buzzsumo permite detectar a aquellos generadores de contenido y los sitios en los que han sido publicados. De igual forma, la plataforma permite emitir informes con información relevante acerca de su autoridad social según varios ítems. Agencias como Ogilvy han recurrido a ella para alcanzar el éxito en sus campañas realizadas para empresas de moda. Por último, herramientas como Right Relevance o Anders Pink son otras alternativas viables que centran su actividad en la investigación de contenido, escucha social, búsqueda de influencers, shares, etc.

\subsection{Modelo PRGS para el análisis de resultado}

Finalmente, una vez realizada la colaboración, los resultados deben ser medidos para poder cuantificar la eficacia y la influencia sobre el público. Para ello, es recomendable cuantificar las respuestas siguiendo el modelo PRGS que recoge datos acerca de la Presencia, la Respuesta, la Generación de contenido y las Sugerencias.

\footnotetext{
${ }^{8} 14$ empresas que te hacen regalos si tienes un klout alto. (16 de junio de 2014). Recuperado de: https://goo.gl/RnHkUx
} 
$<$ resencia

P1 Comunidad

P2 Contenido generado marca
$\mathbf{R}_{\text {espuesta }}$

R1 Me gusta

R2 Comentarios

G1 Contenido generado usuario

eneración

Fuente: IAB España

El Modelo PRGS, implementado por la IAB desde su primer estudio de redes sociales, permite monitorear las marcas de moda y su actividad para con los públicos en función del número de seguidores, la cantidad de post emitidos, el número de me gusta, la cantidad de comentarios y de respuestas, al igual que el número de compartidos o retweets. De igual forma, admite nuevas métricas de relevancia para cuantificar la eficiencia de la firma de moda en los social media.

\section{CONCLUSIONES}

Las relaciones con los influencers son una de las acciones de relaciones públicas 4.0. que más repercusión están obteniendo en el sector de la moda debido a su eficacia a la hora de impactar a los públicos; sin embargo, de entre todas las dificultades que presenta la acción, destaca lo complejo que resulta identificar al prescriptor idóneo para cada campaña. Por ello, el relacionador público debe conocer e implementar las distintas herramientas e instrumentos disponibles para seleccionar y monitorizar su actividad con el objetivo de alcanzar el máximo rendimiento durante el tiempo que dure su relación contractual. Por último, a pesar de encontrarse en un momento de madurez, las relaciones con los influencers deben encaminarse a la profesionalización de su actividad resolviendo cuestiones relacionadas con la remuneración y las tareas asignadas. 
De igual forma, son futuras investigaciones aquellas destinadas a analizar y evaluar la eficacia de las campañas en términos de impacto.

\section{REFERENCIAS BIBLIOGRÁFICAS}

Adecec. (2017). Trabajar con influencers. Fórmulas para una relación eficaz. Recuperado el 10 de julio de 2018 de http://www.adecec.com/pdf/adecec_presenta_la_guia_\%E2\%80\%9Ctraba jar_con_influencers._formulas_para_una_relacion_eficaz $\% E 2 \% 80 \% 9 D$ _p df

Augure. (2015). Estatus y prácticas de las relaciones con influencers. Recuperado el 10 de marzo de 2017 de http://ipmark.com/wpcontent/uploads/2015/06/Informe-de-las-relaciones-con-Influencers-2015Augure.pdf

Capriotti, P. (2013). Planificación Estratégica de la Imagen Corporativa. Málaga: Ariel.

Castelló, A. y Del Pino, C. (2015). La comunicación publicitaria con influencers. Redmarka, 14, pp. 21-50.

Del Río, J. N. (2011). Museos y redes sociales, más allá de la promoción. Redmarka, Año IV, 7, pp. 111-123.

Díaz, L. (2017). Soy Marca: quiero trabajar con Influencers. España: Bresca.

González, G. y Barba, J. J. (2013). La perspectiva autobiográfica de un docente novel sobre los aprendizajes de Educación Física en diferentes niveles educativos. Cultura, 9(8), pp. 171-181.

Illera, A. E. y Monge, S. (2018). La profesionalización de los YouTubers: el caso de Verdeliss y las marcas. Revista Latina de Comunicación Social, 73, pp. 37-54.

Katz, E. y Lazarsfeld, P. (2006). Personal Influence. The part played by people in the flow of mass Comunications. New Jersey: Transaction Publishers, New Brunswick. 
PWC (2016). ¿Moda por un tubo? La relación con el cliente omnicanal en el sector moda en España. Retos y oportunidades'. Recuperado el 10 de julio de 2018 de https://www.pwc.es/es/publicaciones/retail-yconsumo/assets/moda-por-un-tubo-relacion-cliente-omnicanal-sectormoda.pdf

Turiel, H. y Bonaga, C. (2016). Mamá, quiero ser youtuber. Todas las claves para entender el fenómeno que ha venido para quedarse. Barcelona: Planeta.

Viñarás, M. (2013). Estrategias de Comunicación para generar confianza. En Comunicación y Hombre, Noviembre, pp. 58-73.

Violante, N.S. y Laurent, J.M. (2017). Nuevos roles profesionales dentro de la agencia publicitaria en la era digital. Redmarka, Año X, 18, pp. 117-136.

\section{DEL AUTOR}

Eduardo Villena Alarcón es doctor en Comunicación Organizacional por la Universidad de Málaga. Licenciado en periodismo, publicidad y relaciones públicas, actualmente compagina el ejercicio de su profesión con la docencia universitaria. Su línea de investigación versa en torno a la comunicación en el sector de la moda. 